\title{
Estimation des impacts sanitaires futurs de la pollution de l'air dans le monde, en Europe et Île-de-France : le projet A-C HIA
}

\author{
Victoria Likhvar', Didier Hauglustaine1, Patrick Kinney², \\ Augustin Colette ${ }^{3}$, Myrto Valari ${ }^{4}$, Konstandinos Markakis ${ }^{4}$, \\ Mathilde Pascal 5 , Sylvia Medina ${ }^{5}$ \\ 1 Laboratoire des sciences du climat et de l'environnement, CEA / CNRS / \\ Université Saint-Quentin-en-Yvelines, Gif-sur-Yvette \\ 2 Department of Environmental Health Sciences, Columbia University, \\ New York, États-Unis \\ 3 Institut national de l'environnement industriel et des risques (Ineris), \\ Verneuil-en-Halatte \\ 4 Laboratoire de météorologie dynamique, \\ Institut Pierre-Simon Laplace / École Polytechnique, Palaiseau \\ 5 Département santé environnement, Institut de veille sanitaire (InVS), \\ Saint-Maurice
}

didier.hauglustaine@lsce.ipsl.fr

La pollution à l'ozone et aux particules fines est un facteur de risque important et de cause de décès prématurés partout dans le monde. Le projet A-C HIA (2011-2014) a rassemblé des spécialistes de la modélisation de la qualité de l'air, du climat et de l'épidémiologie pour étudier l'évolution future de la pollution et du climat et son impact sur la santé. Ce projet a comparé les bénéfices attendus de différents scénarios de politiques de réduction des émissions polluantes entre 2010, 2030 et 2050, tout en prenant en compte les évolutions climatiques sur la même période aux échelles globale, régionale (Europe) et urbaine (Île-de-France). Le scénario le plus ambitieux en termes de réduction des émissions de polluants permettrait de sauver jusqu'à 1,5 million de personnes chaque année dans le monde dès 2030. Cependant, dès 2050, l'influence négative du changement climatique sur la pollution de l'air contrebalancerait dans certaines zones les effets des politiques visant à améliorer la qualité de l'air. Dans les prochaines années, des bénéfices substantiels pour la santé publique pourraient être obtenus grâce à des stratégies coordonnées de réduction des émissions de gaz à effet de serre et d'amélioration de la qualité de l'air.

$\mathrm{N}$ ous sommes tous exposés à la pollution de l'air et, de ce fait, elle constitue un enjeu de santé publique majeur dans le monde, en Europe et en France (Beelen et al., 2013 ; Hoek et al., 2013 ; Raaschou-Nielsen et al., 2013). D'après l'Organisation mondiale de la santé (OMS), en 2010, 3,22 millions de personnes sont décédées prématurément dans le monde à cause de la pollution par les particules fines et 0,15 million à cause de la pollution à l'ozone (Lozano et al., 2012). Respirer quotidiennement des polluants de l'air, comme les particules fines ou l'ozone, entraine des effets néfastes sur la santé tout au long de la vie (figure 1). La pollution de l'air favorise le développement de maladies chroniques graves, comme les troubles de la reproduction et du développement de l'enfant, les cancers, les maladies cardio-vasculaires et respiratoires, ou certaines pathologies neurologiques. Agir sur la pollution de l'air permettrait d'améliorer la santé et le bien-être de l'ensemble de la population (Lozano et al., 2012 ; Medina et al., 2013).
Le projet A-C HIA a évalué l'influence que des politiques visant à réduire les émissions de polluants atmosphériques tels que l'ozone et les particules fines pourraient avoir sur l'évolution de l'état de santé des populations en 2030 et en 2050 , en prenant en compte l'influence du climat et du changement climatique sur la pollution de l'air. Les liens entre pollution de l'air, climat et santé incitent à développer des stratégies ambitieuses et coordonnées de réduction des émissions des gaz à effet de serre et des polluants de l'air. En effet, des polluants atmosphériques comme l'ozone ou les particules fines influencent la santé mais également le climat en perturbant les rayonnements solaire et/ou infrarouge. Inversement, les perturbations du climat (température, humidité, vents, nuages...) associées à l'augmentation des gaz à effet de serre affectent la formation et la dispersion des polluants. A-C HIA a permis de comparer les bénéfices sanitaires attendus sous différents scénarios de réduction des émissions de polluants dans l'air. Une des réussites de A-C HIA a été 


\section{Abstract}

Future health impact assessment of air pollution at the global, European and Île-de-France scales: the Air Pollution Climate Health Impact Assessment (A-C HIA) project

Ozone and fine particles are current risk factors for premature death all over the globe. In coming decades, substantial improvements in public health may be achieved by reducing air pollution. The overall objective of the A-C HIA project (2011-2014) was to apply state of the art climate, air quality, and health modelling tools to assess future health impacts of $\mathrm{O}_{3}$ and PM2.5 under different scenarios of emissions for the period 2030-2050. A-C HIA created an interdisciplinary team to study the impacts of climate change on health through air quality changes, and to establish longer-term collaborations between communities. This question has been explored at three spatial scales: global, regional (Europe), and urban (Île-de-France). We find that 1.5 millions of cardio-vascular deaths could be delayed each year in 2030 compared to 2010. In Europe, air-pollution-related mortality should decrease in $\mathbf{2 0 3 0}$ compared to 2010. At the finer scale (Île-de-France) we found that the respiratory mortality should increase over the highly populated area of Paris. In the coming years, substantial benefits to public health could be achieved through coordinated strategies to reduce emissions of greenhouse gases and improving air quality.

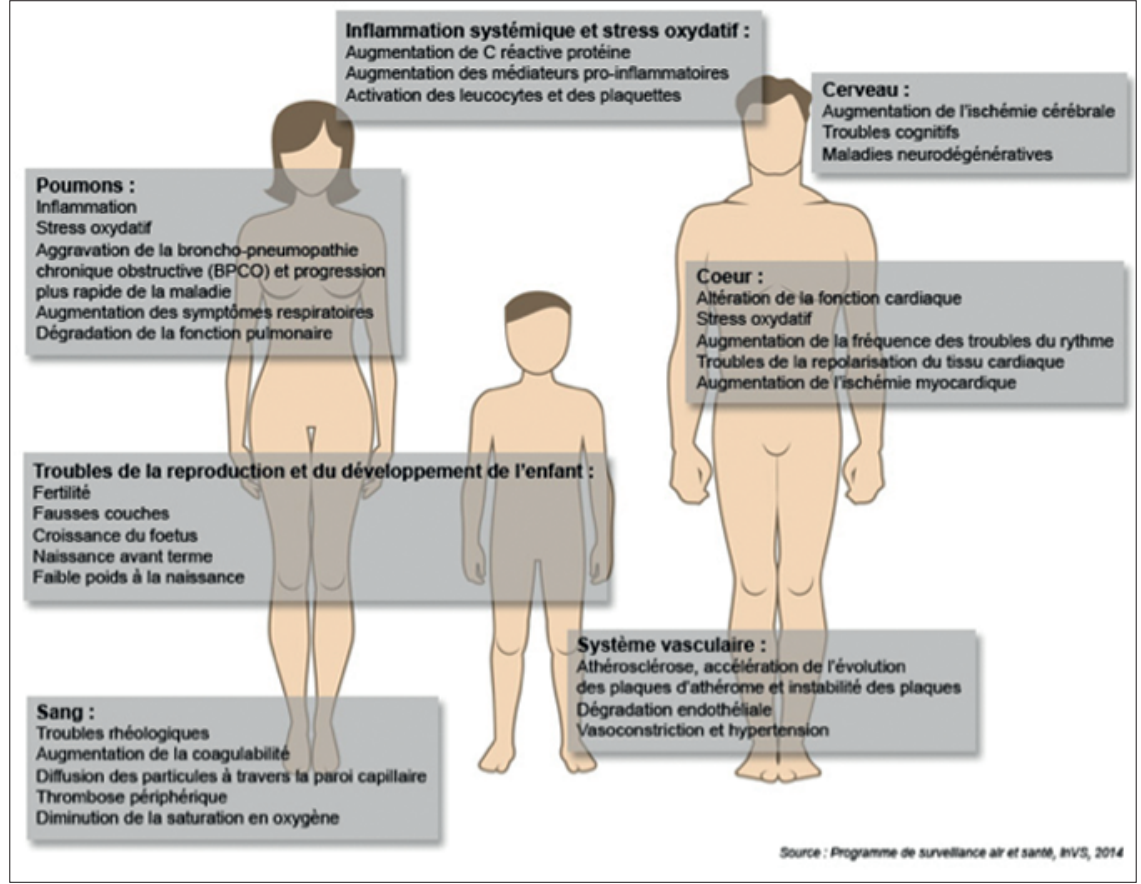

Figure 1. Principaux effets de la pollution de l'air sur la santé. Source : Institut national de veille sanitaire (INVS)

d'interfacer des modèles planétaires, continentaux et régionaux, ce qui permet d'avoir des résultats cohérents et comparables pour le monde, l'Europe et l'Île-de-France (Likhvar et al., 2015).

\section{Comment estime-t-on les impacts sanitaires futurs de la pollution de l'air?}

Le projet A-C HIA a utilisé les outils de modélisation du climat, de la qualité de l'air et de la santé les plus récents (voir encadrés 1 et 2). Il a comparé différents scénarios d'évolution climatique et de réduction des émissions polluantes.

La première étape a consisté à estimer la pollution de l'air en 2030 et 2050 pour le monde, l'Europe et l'île-de-France. Pour cela, il a fallu utiliser des hypothèses d'évolutions des sources de rejets de polluants dans l'air (des scénarios d'émissions polluantes). Le projet a utilisé deux scénarios prospectifs développés par l'International Institute for Applied Systems Analysis (IIASA) dans le cadre du projet européen Eclipse (Stohl et al., 2015) : le premier correspond à l'application de la réglementation actuellement en vigueur et déjà prévue à l'avenir pour la qualité de l'air (scénario CLE, Current LEgislation) et le second à la plus grande réduction d'émissions techniquement faisable (scénario MFR, Maximum Feasible Reduction) par l'implémentation de l'ensemble des solutions technologiques actuellement disponibles pour réduire la pollution (Amann et al., 2013). Ces scénarios couvrent l'ensemble des espèces gazeuses réactives (précurseurs de l'ozone troposphérique et des aérosols : $\mathrm{CH}_{4}, \mathrm{CO}$, $\mathrm{NO} x$, composés organiques volatils ou $\mathrm{COV}, \mathrm{NH}_{3}, \mathrm{SO}_{2}$ ) et particulaires (carbone suie (BC) et carbone organique (OC)) émises à la surface par les activités humaines.

Ces scénarios servent de données d'entrée aux simulations produites par les modèles de chimie atmosphérique LMDz-Inca (Hauglustaine et al., 2014) à l'échelle globale et Chimere (Menut et al., 2013) aux échelles régionale et locale. Ces modèles calculent la concentration des polluants dans l'air à partir des données d'émissions et de données météorologiques. La météorologie joue un rôle très important dans la formation et la dispersion de la pollution de l'air et, à l'inverse, des polluants de l'air comme l'ozone $\left(\mathrm{O}_{3}\right)$ et les particules fines (particulate matter ou PM) ont un impact climatique. Les données météorologiques utilisées par les modèles de chimie atmosphérique sont issues du modèle climatique LMDz (Hourdin et al., 2006). Ce modèle climatique permet de simuler le climat futur en prenant en compte l'évolution des émissions de gaz à effet de serre développée par le Groupe d'experts 
intergouvernemental sur l'évolution du climat (Giec). Le projet A-C HIA a utilisé un scénario moyen de changement climatique, c'est-à-dire ni optimiste ni pessimiste (RCP 4.5.) (Boucher et al., 2015). Il est toutefois à noter que les scénarios Eclipse utilisés pour les polluants réactifs sont plus proches en termes d'émissions de $\mathrm{CO}_{2}$ de la trajectoire suivie par le scénario RCP6.0. Cette limitation a toutefois un impact modéré sur les résultats présentés ici aux horizons 2030 et 2050 .

La deuxième étape a été de transcrire les résultats en impacts sur la santé, en s'appuyant sur des modèles épidémiologiques solides (encadré 2). Nous avons comparé la mortalité observée avec les niveaux actuels de pollution à celle attendue en prenant en compte l'évolution de la qualité de l'air suivant les scénarios décrits brièvement ci-dessus.

\section{Quelles sont les évolutions attendues dans le monde?}

La figure 2 illustre 1'évolution de la concentration annuelle de particules fines de diamètre inférieur à $2,5 \mu \mathrm{m}$ (PM2.5) à la surface en 2030 par rapport à 2010, calculée à l'aide du modèle LMDz-Inca pour les scénarios CLE et MFR, et la figure 3 présente la variation de la surmortalité associée à cette évolution des concentrations.

Aujourd'hui, plus de 2,3 milliards de personnes dans le monde sont exposées à des niveaux moyens de particules fines (PM2.5) supérieurs à ceux recommandés par l'Organisation mondiale de la santé $\left(10 \mu \mathrm{g} / \mathrm{m}^{3}\right)$. Dans le scénario où chaque pays appliquerait la réglementation actuelle sur les émissions, en 2030, plus de 2,8 milliards de personnes resteraient exposées à ces niveaux trop élevés. En Inde, par exemple, le nombre de personnes concernées passerait de plus de 640 millions en 2010 à plus de 1 milliard en 2030. Dans ce scénario, en 2030 , le nombre de décès pour causes cardio-vasculaires liés aux particules fines serait plus élevé qu'aujourd'hui, avec 6800 décès prématurés additionnels par an dans le monde. La majorité de ces décès se produiraient en Asie.

Le scénario traduisant l'implémentation de l'ensemble des technologies de dépollution disponibles conduirait

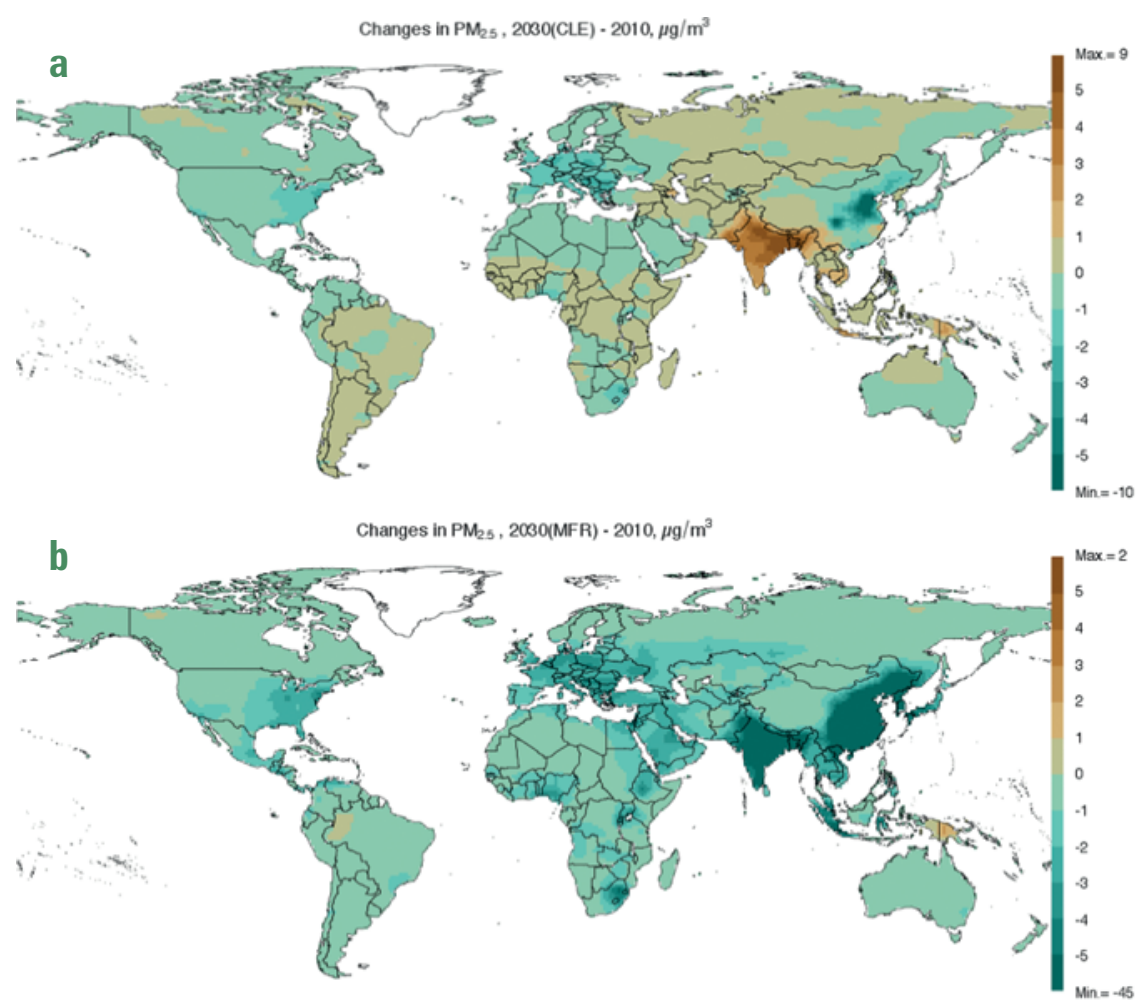

Figure 2. Évolution des concentrations annuelles $\left(\mu \mathrm{g} / \mathrm{m}^{3}\right)$ de particules fines (PM2.5) en 2030 par rapport à 2010, estimée (a) selon le scénario de mise en œuvre des réglementations actuelles et (b) selon le scénario de baisse maximale techniquement faisable.

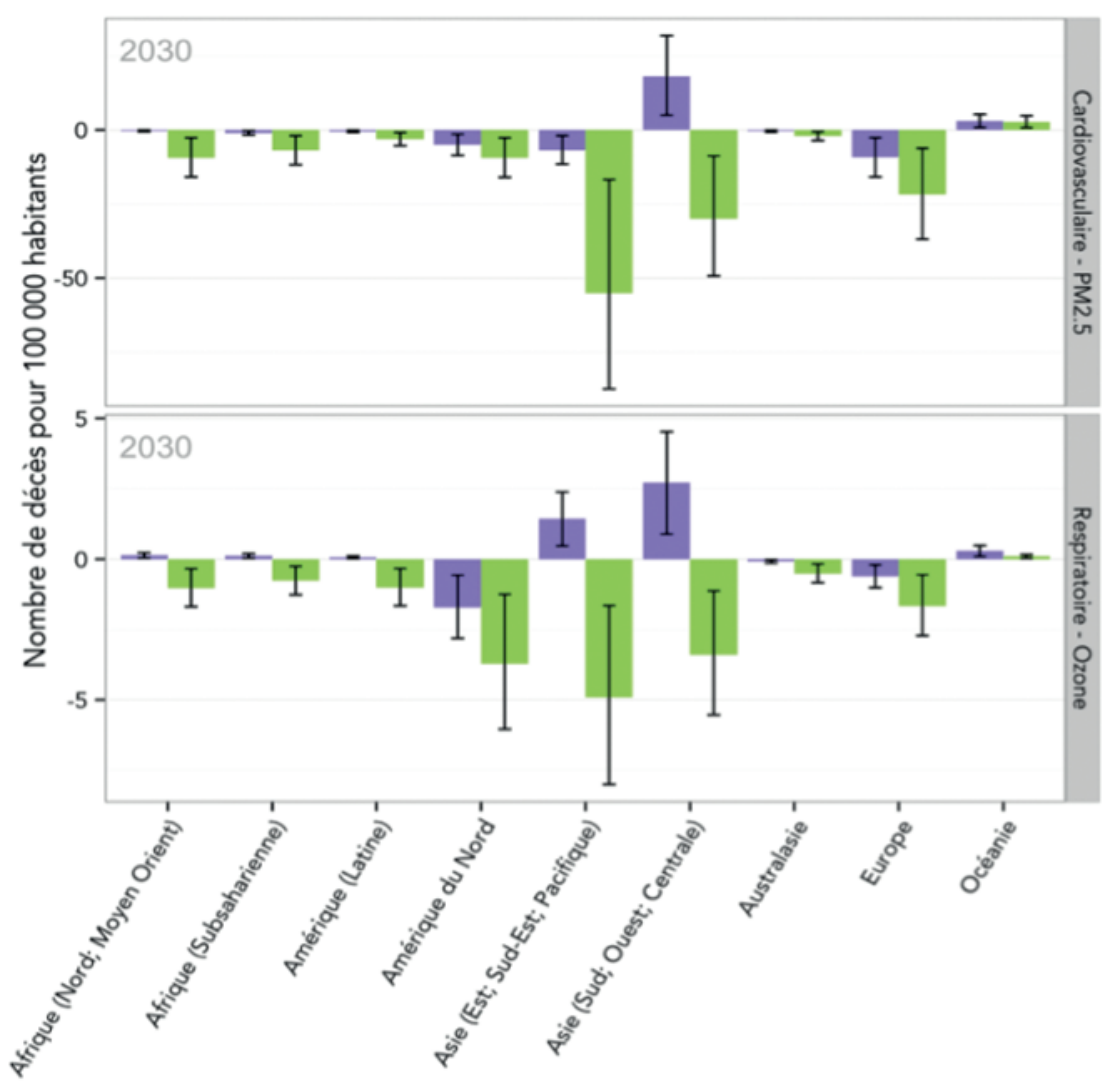

[Réglementation actuelle [ Baisse maximale techniquement faisable

Figure 3. Évolution du nombre de décès (pour 100000 habitants) associée aux évolutions des concentrations de particules fines (en haut) et d'ozone (en bas) par régions du monde en 2030 par rapport à 2010 selon les scénarios de mise en œuvre des réglementations actuelles (en violet) et de baisse maximale techniquement faisable (en vert). 
quant à lui à une baisse de la mortalité. L'Asie est la région qui en bénéficierait le plus, avec des concentrations de PM2.5 qui pourraient être divisées par 4. Ce scénario conduirait également à une amélioration de la qualité de l'air en Amérique du Sud et en Amérique du Nord.

Globalement, si tous les pays du monde mettaient en œuvre l'ensemble des technologies de réduction des émissions connues à ce jour (dans le domaine des transports, du chauffage, de l'industrie, etc.), 1,5 million de décès prématurés pour causes cardio-vasculaires pourraient être évités chaque année.

\section{Quelles sont les évolutions attendues en Europe?}

Le modèle Chimere a été utilisé pour simuler l'évolution des concentrations de polluants à l'échelle de l'Europe. Sur ce continent, une amélioration de la qualité de l'air et de la santé est attendue pour les deux scénarios considérés. En 2010, plus de 250 millions d'Européens étaient exposés à des niveaux de particules supérieurs à la valeur recommandée par l'Organisation mondiale de la santé. Ce nombre pourrait être diminué de moitié en 2030 si la réglementation actuelle était respectée. Ainsi, dès 2030, 109000 décès prématurés pour causes cardio-vasculaires pourraient être évités chaque année (figure 4).

Si les pays mettaient en place toutes les mesures techniques disponibles pour améliorer la qualité de l'air, le bénéfice serait deux fois plus important. Les bénéfices les plus élevés sont attendus en Europe de l'Est pour les PM2.5, et en Europe du Sud pour l'ozone (figure 5).

\section{Quelles sont les évolutions attendues en Île-de-France?}

Pour 1'Île-de-France, nous nous sommes attachés à comparer l'année 2050 à 2010 sur la base des concentrations de polluants simulées par le modèle Chimere à l'échelle locale. À cette échelle, en 2050, le scénario le plus ambitieux pourrait éviter plus de 2800 décès prématurés chaque année (figure 4). Par rapport à 2010, cela représenterait une baisse de la mortalité cardio-vasculaire de près de $20 \%$.

\section{Modélisation de la pollution de l'air aux différentes échelles}

Échelle globale

L'évolution future de la composition de simulée avec le modèle couplé de chimie-aérosols-climat LMDz-INCA couplant en ligne le modèle de circulation générale (MCG) LMDz (Laboratoire de météorologie dynamique, version 5 (Hourdin et al., 2006) et le modèle Inca (Interaction avec la chimie et aérosols, version 4) (Hauglustaine et al., 2014). Dans la configuration actuelle, le modèle comprend 39 niveaux verticaux hybrides. La résolution horizontale est de $1,89^{\circ}$ en latitude et $3,75^{\circ}$ en longitude, ce qui équivaut à environ $200 \mathrm{~km}$ de côté aux latitudes moyennes. Pour une description plus détaillée et une évaluation approfondie du MCG $\mathrm{LMDz}$, nous nous référons à Hourdin et al.. (2006). Inca représente la photochimie de la troposphère incluant les aérosols troposphériques tels que les sulfates, les nitrates, le carbone suie et le carbone organique, ainsi que les aérosols naturels tels que les sels de mer et les poussières désertiques.

\section{Échelle européenne}

Pour modéliser l'impact des émission futures sur la pollution de l'air en Europe à une échelle régionale, nous avons utilisé le modèle de chimie-transport Chimere (Menut et al., 2013) qui calcule le deveni des polluants primaires et secondaires (y compris les aérosols organiques et inorganiques secondaires). Le modèle a été largement utilisé pour des applications de recherche portant sur la qualité de l'ai historique, ainsi que pour la prévision de la qualité de l'air opérationnelle. Les résultats de Chimere en termes $d^{\prime} \mathrm{O}_{3}$ et de particules fines ont étés évalués et comparés à d'autres modèles existants dans le cadre d'exercices d'intercomparaison (Vautard et al., 2009) dont certains axés sur des simulations à long terme (Colette et al., 2012). Nous avon utilisé les sorties de modèles à $0,5^{\circ}(\approx 40 \mathrm{~km})$ I'atmosphère à l'échelle globale a été de résolution couvrant l'Europe. Les conditions aux limites chimiques ont été de la composition chimique sur l'Europe simulée avec le modèle LMDz-Inca.

\section{Île-de-France}

La région île-de-France, située à $1,25-3,58^{\circ} \mathrm{E}$ et à $47,89-49,45^{\circ} \mathrm{N}$, possède une population d'environ 11,7 millions, dont deux millions vivant dans la ville de Paris. La zone est située loin des côtes et se caractérise par une topographie uniforme et faible, ne dépassant pas $200 \mathrm{~m}$ au-dessus du niveau de la mer. Pour modéliser la qualité de l'air dans la région, nous avons utilisé le modèle Chimere à $0,05^{\circ}(\simeq 4 \mathrm{~km})$ de résolution horizontale dans un domaine 156 $\times 128$ km² couvrant l'île-de-France. Les émissions de polluants pour l'île-de-France étaient disponibles à intervalles horaires avec une résolution spatiale de $1 \times 1 \mathrm{~km}^{2}$ (agrégés à $4 \mathrm{~km}$ ). L'ensemble des données sur les émissions a été compilé par des experts locaux en utilisant une variété d'informations spécifiques sur la ville et intégrant un certain nombre d'activités anthropiques dans la région (Airparif, 2012). La répartition spatiale des émissions a été effectuée par sur base des cartes de population à haute résolution, du réseau routier et de l'emplacement des unités industrielles. Les conditions aux limites et initiales pour les simulations sont issues de la simulation à l'échelle européenne du modèle Chimere.

Afin de maintenir la cohérence et de pouvoir comparer les résultats entre les échelles, nous avons harmonisé l'inventaire local en termes de flux annuels d'émissions avec les estimations Eclipse de IIASA (Amman et al., 2013) utilisées dans le modèle européen. Pour fournir des estimations futures des émissions en île-de-France, nous avons utilisé une procédure équivalente (Markakis et al., 2014) obtenues à partir d'une moyenne sur 10 ans

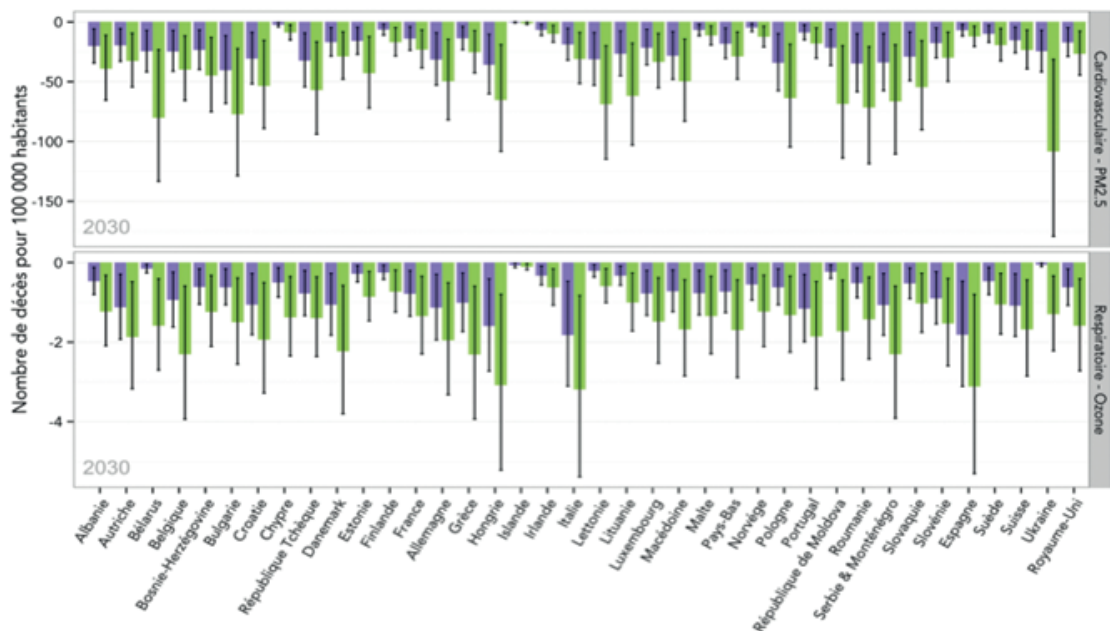

Figure 4. Évolution du nombre de décès (pour 100000 habitants) associée aux évolutions des concentrations de particules fines (en haut) et d'ozone (en bas) pour les différents pays européens en 2030 par rapport à 2010 selon les scénarios de mise en œuvre des réglementations actuelles (en violet) et de baisse maximale techniquement faisable (en vert). 

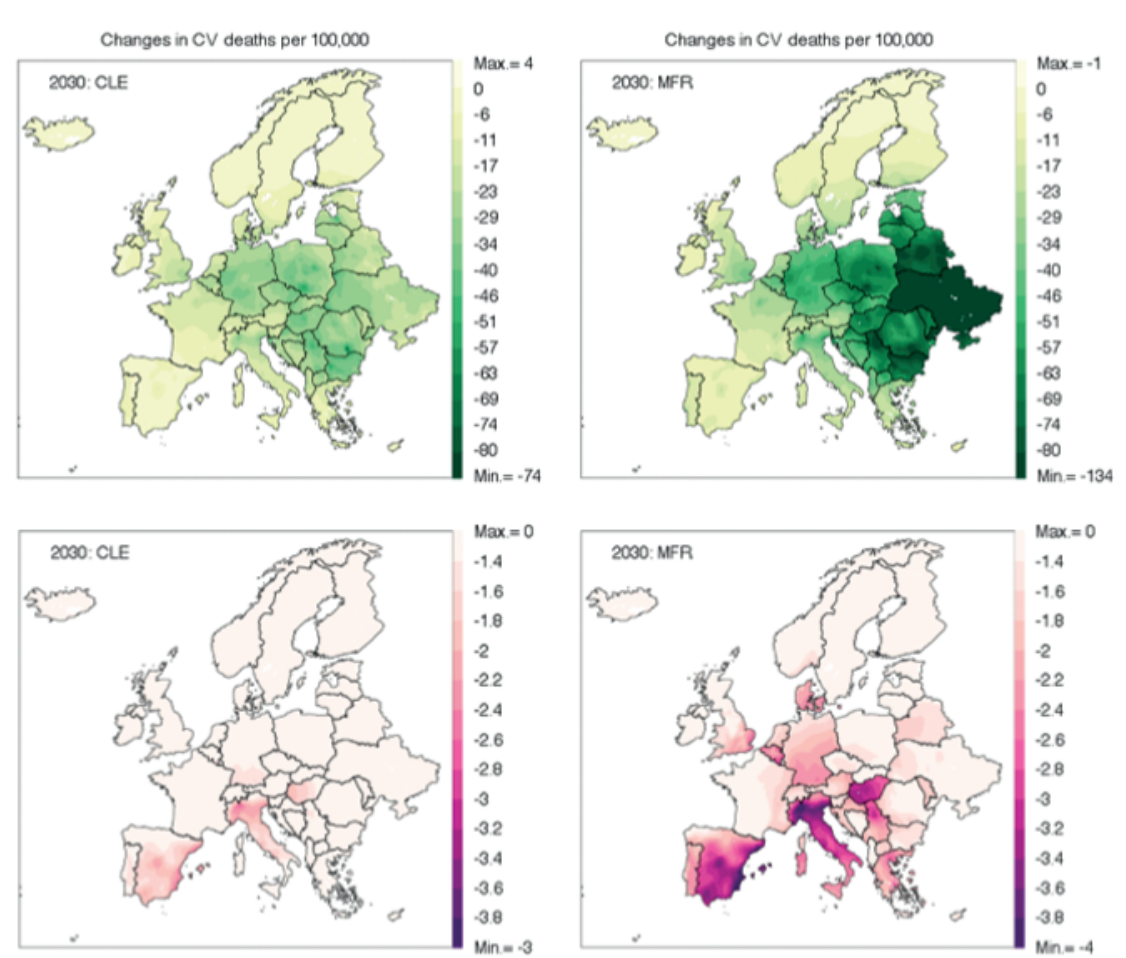

Figure 5. Évolutions du nombre de décès (pour 100000 habitants) associée aux évolutions des concentrations de particules fines (en vert) et d'ozone (en rose) en Europe en 2030 par rapport à 2010, pour les scénarios réglementation actuelle (à gauche) et baisse maximale techniquement faisable (à droite).

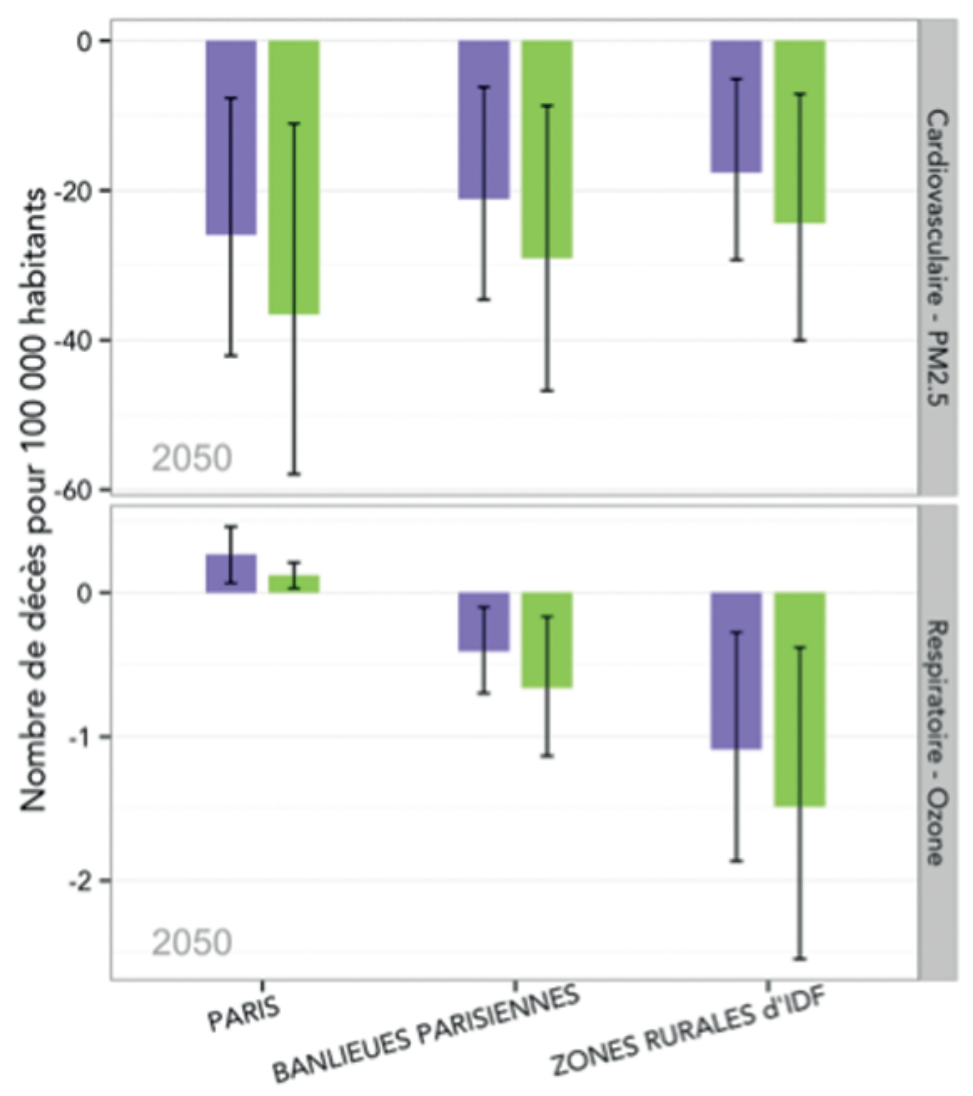

Réglementation actuelle [Baisse maximale techniquement faisable

Figure 6. Évolution du nombre de décès (pour 100000 habitants) associée aux évolutions des concentrations de particules fines (en haut) et d'ozone (en bas) en Île-de-France en 2050 par rapport à 2010 pour les scénarios de mise en œuvre des réglementations actuelles (en violet) et de baisse maximale techniquement faisable (en vert).

\section{Quelles sont les évolutions entre 2030 et 2050 ?}

Dans le monde et en Europe, peu de changements sont observés entre 2030 et 2050 pour les concentrations de PM2.5. En effet, les scénarios font l'hypothèse que la majorité des mesures seraient prises avant 2030 mais que l'effet propre du changement climatique sur les PM2.5 ne devient visible qu'à partir de 2050. À l'inverse, si pour l'ozone on s'attend globalement à une amélioration en 2030, les effets sanitaires défavorables pourraient augmenter à nouveau en 2050. Cette augmentation entre 2030 et 2050 s'explique par la multiplication des situations météorologiques favorables à la formation d'ozone (augmentation des températures et épisodes plus fréquents et plus longs de canicules, stagnation des masses d'air polluées...).

\section{Quelles sont les limites de cette étude?}

Ces résultats sont soumis à des incertitudes, qui sont à la fois liées à l'utilisation de modèles et de scénarios d'émissions. Il n'a en effet pas été possible de prendre finement en compte l'ensemble des facteurs qui pourraient influencer la pollution de l'air dans le futur.

Par exemple, l'accroissement de la population dans les zones urbaines n'a pas été considéré dans ce projet, alors qu'elle se traduirait par une population exposée à la pollution de l'air qui pourrait être beaucoup plus importante en 2030 et 2050 qu'aujourd'hui. L'évolution des décès prématurés effectués plus haut l'est donc à population constante.

L'échelle géographique d'analyse et la disponibilité de données environnementales et sanitaires de bonne qualité jouent également sur les résultats. C'est pourquoi les résultats pour le monde sont jugés plus incertains que ceux pour l'Europe, eux-mêmes plus incertains que les résultats en Île-de-France.

Enfin, le projet ne couvre qu'une partie des impacts de la pollution de l'air sur la santé en se limitant aux décès. La morbidité, baisse de qualité de vie des personnes qui développent une maladie chronique souvent grave liée à la 


\section{Estimation de l'impact sur la santé}

Les concentrations d'ozone et de particules fines simulées aux différentes échelles par les modèles de chimie-transport (encadré 1), ainsi que les données de population et mortalité de référence ont été interpolées sur une même grille géographique. Sur la base de ces résultats et en appliquant des relations concentration-réponse classiques, une surmortalité peut être calculée pour les différents scénarios considérés.

\section{Population et mortalité}

Nous avons utilisé les données de la population mondiale quadrillées, les frontières des pays et des côtes du Center for International Earth Science Information Network (CIESIN)/ Columbia University et Centro Internacional de Agricultura Tropical (CIAT) (2005). Pour éviter un écart entre les estimations de la population maillées et la population de la base de données de mortalité de la WHO (2011), nous avons ajusté les données de population maillées à la population de plus de 15 ans pour l'année 2008. Les chiffres de population par commune d'île-de-France ont été fournis pour l'année 2009 par l'Institut national de la statistique et des études économiques (Insee). À l'échelle mondiale, nous avons obtenu des données de mortalité actuelle pour les 193 pays pour l'année de référence 2008 à partir de la base de données de l'Organisation mondiale de la santé (WHO, 2011) qui recueille les données de mortalité fournies chaque année par les États membres à partir de leurs systèmes d'état civil. Les données extraites contiennent des estimations spécifiques au pays par année, telles que le nombre de décès, le taux de mortalité et le taux de mortalité normalisé selon l'âge pour les deux groupes d'âge (15-59 ans et plus de 60 ans) et sont stratifiées par cause de décès : "toutes causes 》 (CIM-10) cardio-vasculaires (CIM $10:$ 100-199) respiratoire (CIM 10 : J30-J98). Les données européennes de base sont obtenues à partir du même ensemble que les données globales. Les données de mortalité cardio-vasculaire et respiratoire de 1300 communes de la région île-de-France, pour les années 2000-2010, ont été obtenues à partir du Centre d'épidémiologie sur les causes médicales de décès (France). Initialement, pour 2030 et 2050 , nous avons gardé des données de mortalité et de population de

pollution (asthme, cancer du poumon, maladies cardio-vasculaires, etc.), n'a pas été étudiée, car les données nécessaires n'étaient pas disponibles dans l'ensemble des pays du monde.

Signalons par ailleurs que le choix de la relation concentration-réponse est également soumis à des incertitudes. En référence. Dans l'analyse de sensibilité nous avons examiné les changements prévus dans les taux de mortalité de base et de la population pour 2030 dans plusieurs régions du monde, en utilisant les projections de l'Organisation mondiale de la santé (Mathers et Loncar, 2006).

\section{Impact de la pollution sur la santé}

Comme cela a été proposé dans de nombreux travaux (par exemple dans Krewski, 2009), la méthode utilisée pour le calcul de l'impact de l'ozone et des particules fines sur la santé se fonde sur l'évolution des concentrations de ces deux polluants atmosphériques en utilisant une fonction " concentrationréponse » (CRF) obtenue à partir d'études épidémiologiques. Les changements de la mortalité sont estimés en utilisant l'équation suivante :

$$
\Delta Y=Y_{0} \times(1-\exp (-\beta \times \Delta X))
$$

où $\Delta Y$ est l'incidence future sur la santé pour une population donnée par rapport au présent, $Y_{0}$ est la mortalité de base pour une population donnée, $\beta$ est la CRF qui décrit la relation entre le risque (un changement de la concentration du polluant) et le changement correspondant de l'effet sur la santé et $\Delta X$ un changement dans la concentration du polluant.

Considérant les données probantes actuelles sur les impacts sanitaires et la disponibilité des données sur la santé nous avons décidé de nous concentrer sur les impacts à long terme des PM2.5 sur la mortalité cardio-vasculaire et de $\mathrm{I}^{\prime} \mathrm{O}_{3}$ su la mortalité respiratoire. Pour les PM2.5 nous avons utilisé la CRF fournie par une méta-analyse récente (Hoek et al., 2013). Pour l'ozone, nous avons utilisé le risque relatif de maladie respiratoire à partir de Jerrett et al. (2009). Nous avons calculé $\Delta X$ comme la différence entre les concentrations calculées pour l'avenir pour un scénario donné et le concentrations de la période de 2010. I faut noter que la limitation principale des travaux consiste à choisir des relation concentration-réponse déterminées à partir de cohortes spécifiques disponibles aux États-Unis ou en Europe et à les appliquer à l'échelle globale. Nous avons effectué notre analyse au niveau de la cellule de la grille et agrégé les résultats dans le pays ou au niveau régional à des fins de présentation. Les pays et régions ont été choisis en fonction de la classification de l'OMS

effet, les relations couramment utilisées sont fondées sur des cohortes aux États-Unis ou en Europe et ne sont pas nécessairement représentatives de la situation dans les pays en développement. Par ailleurs, ces relations peuvent évoluer dans le futur pour plusieurs raisons (modification $\mathrm{du}$ système de santé, vieillissement de la population...). Cette évolution n'est pas prise en compte dans les travaux actuels. Enfin, d'autres polluants pour lesquels de telles relations ne sont actuellement pas disponibles seraient également à prendre en compte dans le cadre de travaux futurs.

\section{Que veulent dire ces résultats en termes de santé publique?}

Aujourd'hui, la pollution de l'air est un facteur de dégradation de la santé de l'ensemble de la population. Les décideurs s'appuient sur les scénarios d'émissions et les modèles pour définir des politiques visant à améliorer la qualité de l'air. Le projet A-C HIA montre les bénéfices sanitaires associés à ces politiques.

Nous avons étudié deux scénarios d'émissions des polluants de l'air et un scénario d'évolution climatique. Les résultats indiquent que des bénéfices sanitaires très importants seraient obtenus en mettant en place le scénario le plus ambitieux de réduction des émissions. Ces bénéfices seraient partagés par beaucoup de pays dans le monde et se manifesteraient également en France et en particulier en Île-de-France.

Ces bénéfices sont une estimation a minima, puisque le projet n'a pas pris en compte les impacts positifs que l'amélioration de la qualité de l'air aurait sur le développement de maladies, le recours aux soins médicaux et la qualité de vie.

La mise en place de l'ensemble des technologies disponibles pour réduire les émissions de différents secteurs d'activité sera difficile et coûteuse à mettre en place. Mais les efforts nécessaires doivent toutefois être mis en perspective avec les bénéfices sanitaires et économiques potentiellement associés.

Il faut également souligner que les scénarios considérés ici ne prennent en compte que des mesures technologiques et n'envisagent pas des modifications de comportement et de consommation. Or, les changements de comportement, comme le développement de modes de transport actif comme le vélo ou la marche, ou l'usage des transports en communs, contribuent également à 
améliorer la qualité de l'air, tout en favorisant la santé et le bien-être.

Dans un contexte de changement climatique, il est crucial que les politiques de réduction des émissions de polluants de l'air et de gaz à effet de serre soient coordonnées. Ceci permettra d'être plus efficace dans l'amélioration de la qualité de l'air à court terme tout en limitant les effets négatifs du changement climatique à plus long terme

\section{Remerciements}

Le projet A-C HIA a été soutenu par le GIS Climat-Environnement-Société et financé par l'Ademe, et a été partiellement financé par la Commission européenne dans le cadre du projet FP7 Hikari sous le contrat numéro 313987. Les simulations numériques ont été effectuées sur les ordinateurs du TGCC/CCRT dans le cadre d'allocations du GENCI.

\section{Bibliographie}

Airparif, 2012. Évaluation prospective des émissions et des concentrations des polluants atmosphériques à I'horizon 2020 en île-de-France - Gain sur les émissions en 2015. Rapport disponible à : www.airparif.asso.fr/_pdf/publications/ppa-rapport-121119.pdf

Amman M., Klimont Z., Wagner F., 2013. Regional and global emissions of air pollutants: recent trends and future scenarios. Annu. Rev. Environ. Resources, 38, 31-55

Beelen R. et al., 2013. Effects of long-term exposure to air pollution on natural-cause mortality: an analysis of 22 European cohorts within the multicentre ESCAPE project.The Lancet, 383, 785-795.

Boucher 0., Dufresne J.-L., Vial J., Brun E., Cattiaux J., Chauvin F., Salas y Mélia D., Voldoire A., Bopp L., Braconnot P., Ciais P., Yiou P., Guilyardi E., Mignot J., Guivarch G., 2015. Projection des changements climatiques futurs. La Météorologie, 88, 56-68.

Center for International Earth Science Information Network (CIESIN)/Columbia University et Centro Internacional de Agricultura Tropical (CIAT). 2005. Gridded Population of the World, Version 3 (GPWv3): Population density grid. Palisades, NY. [consulté le 23 janvier 2013]. Disponible à : http://sedac.ciesin.columbia.edu/data/set/ gpw-v3-population-density

Colette A., Granier C., Hodnebrog O., Jakobs H., Maurizi A., Nyiri A., Rao S., Amann M., Bessagnet B., D'Angiola A., Gauss M., Heyes C., Klimont Z., Meleux F., Memmesheimer M., Mieville A., Rouill L., Russo F., Schucht S., Simpson D., Stordal F., Tampieri F., Vrac, M., 2012. Future air quality in Europe: a multi-model assessment of projected exposure to ozone. Atmos. Chem. Phys., 12, 10613-10630.

Hauglustaine D.A., Balkanski Y., Schulz M., 2014. A global model simulation of present and future nitrate aerosols and their direct radiative forcing of climate. Atmos. Chem. Phys., 14, 11031-11063.

Hoek G., Krishnan R.M., Beelen R., Peters A., Ostro B., Brunekreef B., Kaufman J.D., 2013. Long-term air pollution exposure and cardio-respiratory mortality: a review. Environ Health, 12, 43-54.

Hourdin F., Musat I., Bony S., Braconnot P., Codron F., Dufresne J.-L., Fairhead L., Filiberti M.-A., Friedlingstein P., Grandpeix J.-Y., Krinner G., Levan P., Li Z.-X., Lott F., 2006. The LMDZ4 general circulation model: climate performance and sensitivity to parametrized physics with emphasis on tropical convection. Clim. Dyn., 27, 787-813.

Jerrett M., Burnett R.T., Pope C.A., Ito K., Thurston G., Krewski D., Shi Y., Calle E., Thun M., 2009. Long-term ozone exposure and mortality. N. Engl. J. Med., 360, 1085-1095.

Krewski D., 2009. Evaluating the effects of ambient air pollution on life expectancy. N. Engl. J. Med., 360, 413-415.

Likhvar V., Pascal M., Markakis K., Colette A., Hauglustaine D., Valari M., Klimont Z., Medina S., Kinney P., 2015. A multi-scale health impact assessment of air pollution over the 21 st Century in a changing climate. Sci. Total Environ., 514, 439-449.

Lozano R., Naghavi M., Foreman K., Lim S., Shibuya K., Aboyans V., Abraham J., Adair T., Aggarwal R., Ahn S. Y., Alvarado M., Anderson H. R., Anderson L. M., Andrews K. G., Atkinson C., Baddour L. M., Barker-Collo S., Bartels D. H., Bell M. L., Benjamin E. J., Bennett D., Bhalla K., Bikbov B., Bin A. A., Birbeck G., Blyth F., 2012. Global and regional mortality from 235 causes of death for 20 age groups in 1990 and 2010: a systematic analysis for the global burden of disease study 2010. The Lancet, 380, 2095-2128.

Markakis K., Valari M., Colette A., Sanchez O., Perrussel O., Honore C., Vautard R., Klimont Z., Rao S., 2014. Air-quality in the mid-21st century for the city of Paris under two climate scenarios; from regional to local scale. Atmos. Chem. Phys., 14, 7323-7340.

Mathers C.D., Loncar D., 2006. Projections of global mortality and burden of disease from 2002 to 2030. PLOS Med., 3. doi: 10.1371/journal.pmed.0030442

Medina S., Ballester F., Chanel O., Declercq C., Pascal M., 2013. Quantifying the health impacts of outdoor air pollution: useful estimations for public health action. J. Epidemiol. Commun. Health, 67, 480-483.

Menut L., Bessagnet B., Khvorostyanov D., Beekmann M., Blond N., Colette A., Coll I., Curci G., Foret G., Hodzic A., Mailler S., Meleux F., Monge J.-L., Pison I., Siour G., Turquety S., Valari M., Vautard R., Vivanco M.G., 2013. Chimere 2013: a model for regional atmospheric composition modelling. Geosci. Model Dev., 6, 981-1028. doi: 10.5194/gmd-6-981-2013

Raaschou-Nielsen O., Andersen Z.J., Beelen R., Samoli E., Stafoggia M., Weinmayr G., Hoffmann B., Fischer P., Nieuwenhuijsen M.J., Brunekreef B., Xun W.W., Katsouyanni K., Dimakopoulou K., Sommar J., Forsberg B., Modig L., Oudin A., 2013. Air pollution and lung cancer incidence in 17 European cohorts: prospective analyses from the European study of cohorts for air pollution effects (Escape). Lancet Oncol., 14, 813-822.

Stohl A., Aamaas B., Amann M., Baker L. H., Bellouin N., Berntsen T. K., Boucher O., Cherian R., Collins W., Daskalakis N., Dusinska M., Eckhardt S., Fuglestvedt J. S., Harju M., Heyes C., Hodnebrog Ø., Hao J., Im U., Kanakidou M., Klimont Z., Kupiainen K., Law K. S., Lund M. T., Maas R., Maclntosh C. R., Myhre G., Myriokefalitakis S., Olivié D., Quaas J., Quennehen B., Raut J.-C., Rumbold S. T., Samset B. H., Schulz M., Seland Ø., Shine K. P., Skeie R. B., Wang S., Yttri K. E., Zhu T., 2015. Evaluating the climate and air quality impacts of short-lived pollutants. Atmos. Chem. Phys. , 15, 10529-10566. doi: 10.5194/acp-15-10529-2015

Vautard R., Schaap M., Bergström R., Bessagnet B., Brandt J., Builtjes P.J.H., Christensen J.H., Cuvelier C., Foltescu V., Graff A., Kerschbaumer A., Krol M., Roberts P., Rouill L., Stern R., Tarrason L., Thunis P., Vignati E., Wind P., 2009. Skill and uncertainty of a regional air quality model ensemble. Atmos. Environ., 43, 4822-4832.

WHO, 2011. WHO mortality data base documentation. World health Organisation Health statistics and health information systems. [consulté le 18 décembre 2012]. Disponible à www.who.int/healthinfo/ global_burden_disease/projections/en/index.html 